\title{
Analysis of swallowing movement using a simple and safe device
}

\author{
Masamichi Takagi 1), Yozo Miyaoka ${ }^{2)}$, Keiko Haishima ${ }^{3)}$, Hiroyuki Haishima ${ }^{3)}$, \\ Kazuhide Matsunaga ${ }^{4)}$ and Yoshiaki Yamada ${ }^{5)}$ \\ "Division of Pediatric Dentistry, Niigata University Graduate School of Medical and Dental Sciences, \\ ${ }^{2}$ Department of Health and Nutrition, School of Health Sciences, Niigata University of Health and Welfare, \\ ${ }^{3)}$ Department of Hygiene and Oral Health, School of Dentistry, Showa University, \\ 4) Division of Maxillofacial Diagnostic and Surgical Sciences, Faculty of Dental Science, Kyushu University, \\ 5) Division of Oral Physiology, Niigata University Graduate School of Medical and Dental Sciences
}

[Recieved: May 16, 2001.]

Key Word: swallowing, laryngeal movement, safe device

\begin{abstract}
The present study reports a newly developed laryngeal movement tracking system. The system consists of a metal rod, a displacement sensor, a plastic plate, and two identical magnets. The plastic plate was fixed to the skin over laryngeal prominence. The rod and magnets conduct the movement of the plate in relation to laryngeal movement to the sensor during swallowing. To evaluate the performance of the system, sensor outputs during swallowing were compared with recordings of masseter and digastric muscle EMGs in 6 young and healthy adults. Tests were conducted both in head-fixed $(n=3)$ and head-free $(n=3)$ conditions. Each subject was given a test fluid $(2.0$ $\mathrm{ml}$ of tea, at a temperature of $25{ }^{\circ} \mathrm{C}$ ) into the oral cavity and was instructed to swallow it. Actual motion picture of the laryngeal movement during swallowing was also recorded in the sagittal plane using a video tape recorder in one subject. The swallowing event in the laryngeal movement could be recorded as a biphasic curve by the system both in head-fixed and head-free conditions. Temporal analysis of the recorded curve together with the EMGs and the motion picture revealed that; the recorded curve reflects natural movement of the larynx during swallowing; and the inflections on the curve corresponded to laryngeal upward and downward movements respectively. The present results suggest that the developed system may be applicable to obtain temporal aspects of swallowing easily and safely in the clinic and laboratory.
\end{abstract}




\section{Introduction}

Practical methods to record swallowing events have been sought for clinical evaluation of dysphagic patients and for swallow research. ${ }^{1)}$ In fact, when we had considered foods for dysphagia patients, we realized the need to know the timing of swallow events. Several methods have been adopted to analyze swallowing events, such as pharyngeal manometer, electroglottograph, videofluoroscopy, ausculation, electromyography (EMG) of swallow-related muscles, and functional magnetic resonance imaging. ${ }^{2-7)}$ However, each has disadvantages and limitations. For example, using of an $\mathrm{X}$ ray apparatus in healthy subjects is unethical, and laryngeal sounds and EMG recordings seem to be difficult to interpret into swallowing events. On the other hand, recording of laryngeal movements with swallow-related muscle activities may give a timing of the swallow-initiation. ${ }^{8)}$ Therefore, we sought a method to measure laryngeal movements using a system, which is simple, easy to handle and reliable.

\section{Materials and methods}

As shown in Fig. 1, the developed laryngeal movement tracking system consisted of a metal rod $(0.5 \mathrm{~mm}$ of diameter, made of elgiloy), two small magnets (concaved, $5.2 \mathrm{~mm}$ of diameter and $2.5 \mathrm{~mm}$ of thickness), a thin plastic plate (12 $\mathrm{mm}$ of diameter), and a displacement sensor (Nihonkohden, TB-611T). The plastic plate was placed on the skin over the laryngeal prominence using an adhesive tape. The displacement sensor detected movement of the plate after swallowing. To conduct the movement of the plate to the sensor, the metal rod and magnets were employed. One magnet was fixed using dental resin to the plate allowing the reverse side to face the skin. The other magnet was fixed to a one end of the rod. The other end of the rod was connected to the displacement sensor. The magnetic poles were arranged to attract each other and contacted. The free magnetic joint helped the rod to transfer the movement of the plate to the sensor smoothly, so that the system did not disturb natural swallowing. When the larynx moved during swallowing, the laryngeal movement was transmitted to the sensor by the device and recorded as a two dimensional wave. The total weight of the system was about $0.9 \mathrm{~g}$.

The performance of the laryngeal movement tracking system was tested in two conditions; head-fixed and head-free conditions. Three young healthy adults (two males and a female) were selected for the head-fixed session, and three young healthy adults (a male and two females) were selected

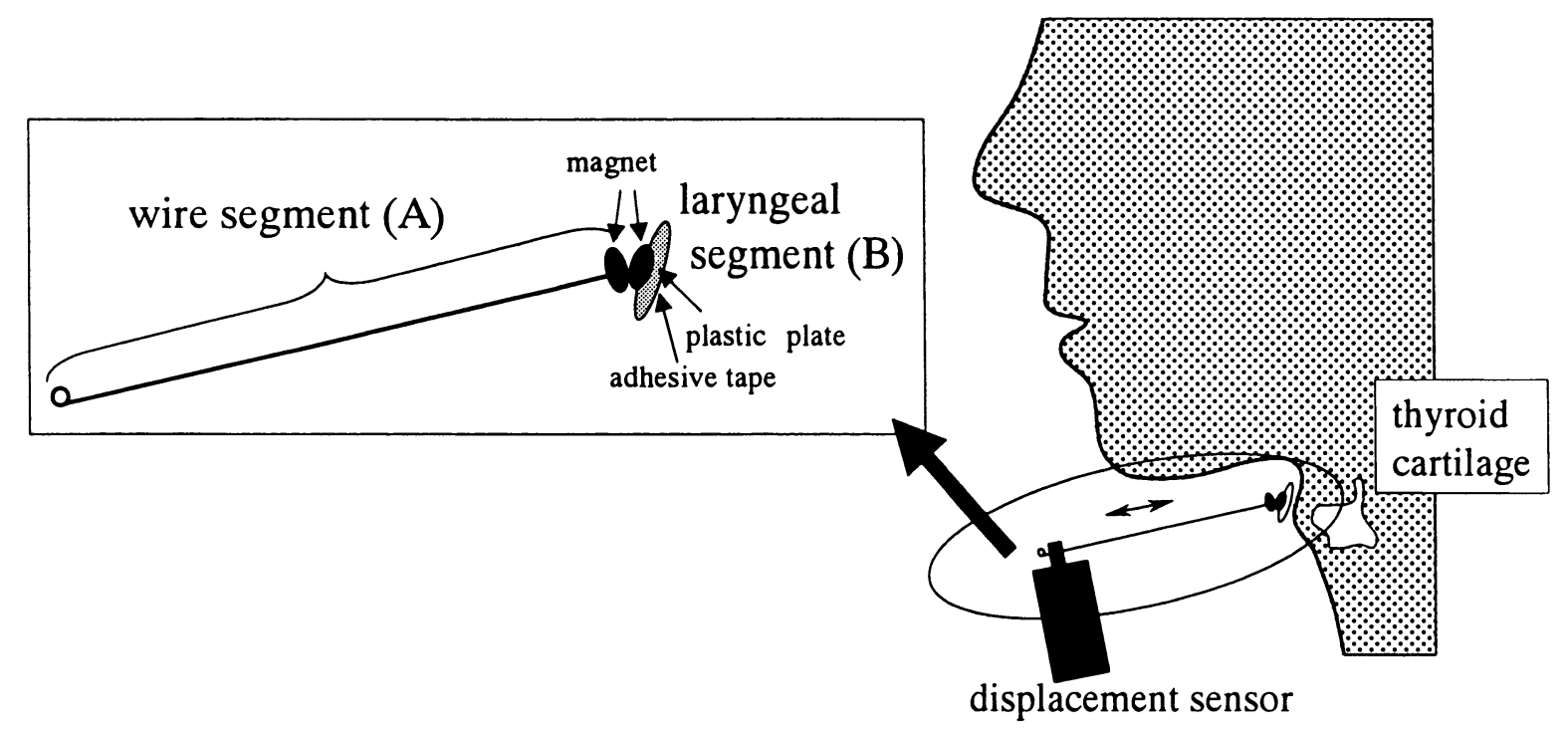

Fig. 1 Setup of developed device

The developed device consists of laryngeal and wire segments. The laryngeal segment ( $\mathrm{A}$ in Fig. 1) is placed on the skin using an adhesive tape, and the wire segment ( $\mathrm{B}$ in Fig. 1) is connected to a displacement sensor Each segment is attracted by the magnetic force. The laryngeal segment is moved with changes in the position of the cartilage during swallowing. This movement is transmitted by the wire and recorded as a "laryngeal 
for the head-free session. All the subjects were explained about the aim and procedure of the experiments. The subject was made to sit on a chair, and the displacement sensor was fixed in front of the subject with a swivel locking apparatus, perpendicular to the mandibular plane (Fig. 1). In the headfixed session, the head of the subject was stabilized with a holder that was fixed to the chair. When the head was placed in the holder, care was taken not to disturb the natural swallowing or to cause any discomfort to the subject.

Electromyograms (EMGs) of the masseter and suprahyoid muscles were obtained from the habitual working-side of the individual subjects with bipolar surface electrodes. The EMGs were amplified, filtered $(30-3,000 \mathrm{~Hz}$ ), and full-rectified. The recorded EMGs and laryngeal movements (measured by the system) were stored on a magnetic tape (TEAC, MR-30) along with a signal that was generated by the operator when he/she indicated the subject to swallow after completion of the test fluid injection.

To elucidate the relation between the obtained displacement (named laryngeal movement curve: LMC) and the actu- al laryngeal movement, video recording was performed as with the recording with the developed system. A video camera (SONY, DCR-PC10) was placed about $1 \mathrm{~m}$ away, at the right side of the subject. The recorded movie was captured and stored with a personal computer. The laryngeal movement was traced on the motion picture obtained from the movie as movement trajectories of the point where the two magnets contacted.

To elicit swallows, test fluid $\left(2.0 \mathrm{ml}\right.$ of tea, at $\left.25^{\circ} \mathrm{C}\right)$ was poured into the floor of the mouth from a $10-\mathrm{ml}$ needle-less syringe. Five trials were conducted in one session. Two or more sessions were performed in each subject with an interval of about 2-weeks.

\section{Results}

Fig. 2 shows a typical laryngeal movement curve (LMC) recorded by the developed system and suprahyoid muscle activities during swallowing in the head-fixed condition. Although masseter muscle activity was recorded with the suprahyoid muscle, its activity was so small that masseter

\section{jaw opening swallowing}

\section{suprahyoid muscles \\ laryngeal movement curve (LMC)}

\section{Signal for swallowing}
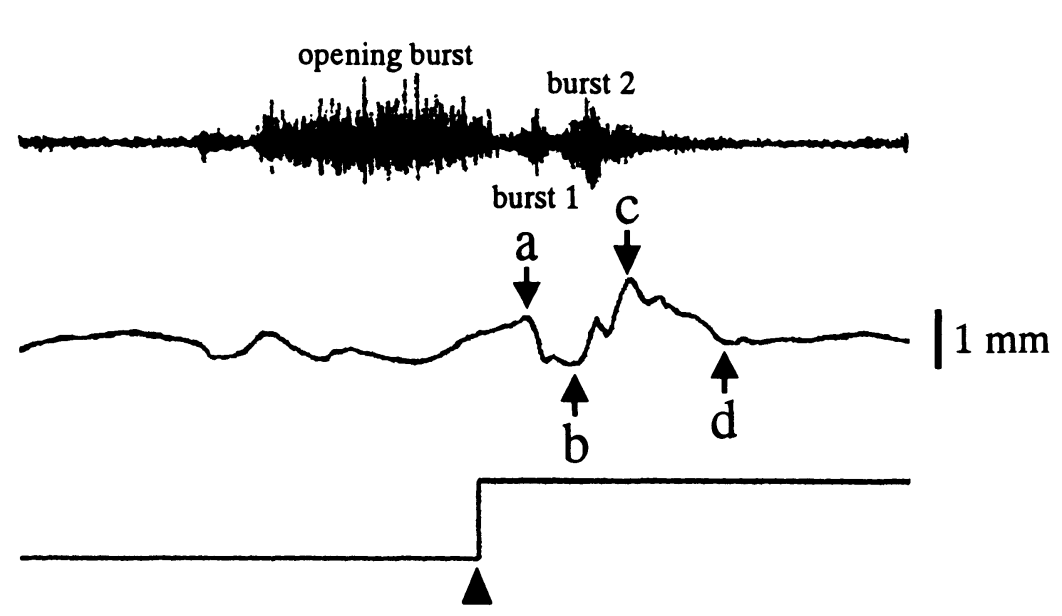

S

Fig. 2 Typical pattern of LMC (laryngeal movement curve) in the head-fixed condition The LMC (middle trace) is always observed with the bursts of the suprahyoid muscles (upper trace) during jaw opening and swallowing. The recorded curve during swallowing consists of three phases: (1) the trajectory of the curve falls down from the baseline when the larynx elevates at the beginning of swallowing (a-b), (2) the trajectory conversely goes up when the elevated larynx falls down (b-c), and (3) the trajectory falls down again and return to the base line when the larynx returns to the rest position (c-d). S: the point at which test food was delivered to the subject and start swallowing. 


\section{The laryngeal movement curve (LMC)}

\section{The actual movement of the larynx (sagittal view)}

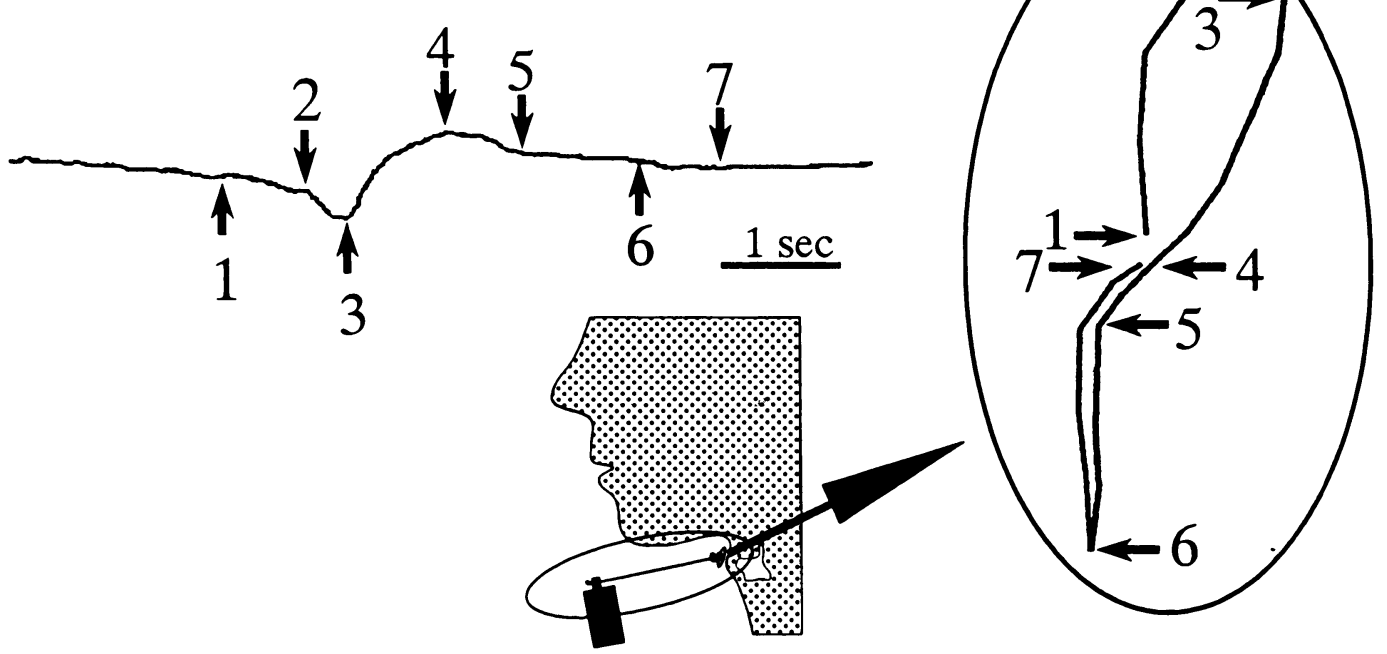

Fig. 3 The relationship between the LMC and actual laryngeal movement during swallowing in the head-fixed condition

Numeral in the laryngeal movement curve (left) and those in the filmed laryngeal movement (right) correspond to each other, but intervals between the serial numbers are not equal. See the text for details.

activity was excluded from the analysis. There were three bursts in the suprahyoid muscle. This was consistent among the subjects tested. The first burst was longer in duration than the other two bursts. The first burst coincided in time with the mouth opening to accept the test fluid. We named the first burst as "opening burst." The opening burst ended when swallowing command was delivered by the operator, which was recognized with the signal shown in the bottom trace. The remaining two bursts coincided in time with biphasic LMC indicated by ' $a$ ', ' $b$ ' and ' $c$ ' on the second trace in Fig. 2 . The onset of the second burst preceded the beginning of laryngeal movement indicated by ' $a$ '. The duration between ' $a$ ' and ' $b$ ' was measured as $1.9 \pm 0.5 \mathrm{sec}$ (mean of the means $\pm \mathrm{SEM}$, $n=3$ ). The sequential events in the recordings were summarized as follows; ${ }^{1)}$ when the subject opened the mouth to accept the test fluid, ongoing activity was appeared in the suprahyoid muscle and the opening movement affected in the LMC starting with sudden downward movement ('a' - 'b' in Fig. 2) and ended with slow recovery to the original position (resting position) ('c' - 'd' in Fig. 2).
In order to confirm the relationship between the actual laryngeal movement and obtained LMC, they were compared in the same time scale. A typical recording in the head-fixed condition was shown in Fig. 3. The numbers (1-7) on the LMC and on the trace obtained from the video recorder corresponded each other in time during swallowing. From the video data, the laryngeal movement was read to start at the point 1 and end at the point 7 , in which ' $a$ ', ' $b$ ', and ' $c$ ' in the LMC were corresponding to points 1,3 , and 4 respectively.

The laryngeal movement tracking system was also tested with the 3 subjects in the head-free condition. According to the temporal relations of the actual laryngeal movement, the obtained LMC was basically the same both in the head-free and head-fixed sessions. However, the baseline of the curve fluctuated more in the head-free condition than in the headfixed condition due to moving of the head. Nevertheless, it was possible to determine the time at which subject started swallowing on the LMC. 


\section{Discussion}

The present study had two objectives; ${ }^{1)}$ to design a system for easy and safe recording of swallowing events; and ${ }^{2)}$ to determine the analytical method to interpret the electrical signal generated by the system into the laryngeal movement.

The first objective was fulfilled. We could temporally capture the swallowing events as a laryngeal movement curve (LMC) using the developed system. The reliability of the LMC during swallowing was confirmed by comparing the LMC with the motion picture of the actual movement of skin over the larynx recorded simultaneously on a video recorder. The recorded LMCs were slightly varied among individuals. The main reason for the variation could be attributed to the individual differences in the form and size of the larynx. ${ }^{9)}$ However, biphasic feature in the LMC during swallowing event was always observed during swallowing in this study. Therefore, the biphasic feature in the LMC could be considered as the normal swallowing pattern.

There are five advantages of the measuring system developed: 1) it is simple (Fig. 1), light in weight (only 0.9 g), and easy to handle, 2) it is easier to operate than the other systems, 3 ) it is safer than videofluoroscopy, 4) it does not disturb any events of swallowing, and 5) it can be assembled with materials, which are ubiquitous and less expensive.

After practically testing the system, we realized the importance of setting the components of the system. The larynx may move upward and forward during swallowing (reference). The system captured the two-dimensional movement of the plastic plate in one-dimension by a displacement sensor. Also the plastic plate and the sensor were connected by a rod. Therefore, the position where the plastic was set and the angle between the rod and the axis of the larynx might affect the recording. The best position for the plastic plate may be slightly rostral to the apex of the laryngeal prominence. The position was found to enable the sensor to detect the onset of the laryngeal movement effectively. The best angle may be perpendicular to each other. The care in setting the system components may be important especially in the subjects with a small larynx or in females. The limitation with female subjects has been noticed when the laryngeal movement was subject to record (reference).

Three inflection points ('a' through 'c' in Fig. 2) were consistently recognized in the LMC following the laryngeal movement during swallowing in all the subjects tested ${ }^{8)}$. After comparing the simultaneous recordings of the suprahy- oid muscle activity and the actual movement of the laryngeal prominence, functional meanings of the three inflection points were sought. Then the point ' $a$ ' in Fig. 2 was determined to coincide in time with the beginning of the laryngeal movement during swallowing. In the same way, the point ' $b$ ' was determined to coincide in time with the laryngeal closure. In fact, the duration between ' $a$ ' and ' $b$ ' was measured as $1.9 \pm$ $0.5 \mathrm{sec}$ (mean of the means $\pm \mathrm{SEM}, \mathrm{n}=3$ ), and coincided with that reported by Preiksaitis et al. ${ }^{9)}$ as the apnoeic period (0.6-2 s) associated with swallows.

EMG of muscles involved in swallowing can provide information on the timing and relative amplitude of selected muscle contraction during swallowing (see Logemann ${ }^{1)}$ for review). In the present experiment, the suprahyoid EMG showed two bursts during swallowing (see bursts 1 and 2 in Fig. 2). These bursts were appeared concomitant with the biphasic feature of the LMC. Anatomically, the suprahyoid muscles include digastric, styloglossal, geniohyoid, and mylohyoid muscles. These muscles terminate at the upper edge of the hyoid bone. Upward and forward pulling of the hyoid-larynx complex is one of the important functions of the suprahyoid muscles during swallowing. Similarly, Ertekin et al. ${ }^{8)}$ recorded two bursts of the supramental muscle activity after voluntarily-induced swallows.

Considering the functions of suprahyoid muscles and the location of the electrodes, the second burst during swallowing may be the activity to lift the hyoid bone. In addition, it was suggested that the first burst during swallowing appeared in concomitant with the movement of the tongue transferring the bolus from oral cavity to pharynx. We believe that using the new system together with the EMG recording is the best way to analyze temporal aspects of swallowing.

\section{References}

1) Logemann, J. A.: Non-imaging techniques for the study of swallowing, Acta Otorhinolaryngol Belg, 48: 139-42, 1994.

2 ) McConnel, F. M., Guffin, T. N., Jr., Cerenko, D. et al.: The effects of bolus flow on vertical pharyngeal pressure measurement in the pharyngoesophageal segment: clinical significance, Otolaryngol Head Neck Surg, 106: 169-74, 1992

3 ) Perlman, A. L. and Grayhack, J. P.: Use of the electroglottograph for measurement of temporal aspects of the swallow: preliminary observations, Dysphagia, 6: 
88-93, 1991.

4 ) Haishima, H., Haishima, K., Taguchi, Y. et al.: Observation of nine-month-old infant's swallowing with digital fluorographic system (X-ray TV system), Dent Jpn, 35: 100-104, 1999.

5 ) Smith, D., Hamlet, S. and Jones, L.: Acoustic technique for determining timing of velopharyngeal closure in swallowing, Dysphagia, 5: 142-6, 1990.

6 ) Murray, K. A., Larson, C. R. and Logemann, J. A.: Electromyographic response of the labial muscles during normal liquid swallows using a spoon, a straw, and a cup, Dysphagia, 13: 160-6, 1998.
7 ) Hamdy, S., Rothwell, J. C., Brooks, D. J. et al.: Identification of the cerebral loci processing human swallowing with $\mathrm{H} 2(15) \mathrm{O}$ PET activation, J Neurophysiol, 81: 1917-26, 1999.

8 ) Ertekin, C., Kiylioglu, N., Tarlaci, S. et al.: Voluntary and reflex influences on the initiation of swallowing reflex in man, Dysphagia, 16: 40-47, 2001.

9 ) Preiksaitis, H. G., Mayrand, S., Robins, K. et al.: Coordination of respiration and swallowing: effect of bolus volume in normal adults, Am J of Physiol, 263: R624-30, 1992. 